\title{
Clinical recommendations for use of lidocaine lubricant during bowel care after spinal cord injury prolong care routines and worsen autonomic dysreflexia: results from a randomised clinical trial
}

\author{
Vera-Ellen M. Lucci ${ }^{1,2} \cdot$ Maureen S. McGrath ${ }^{1,2} \cdot$ Jessica A. Inskip ${ }^{1,2} \cdot$ Shirromi Sarveswaran $^{1} \cdot$ Rhonda Willms $^{2,3,4}$. \\ Victoria E. Claydon ${ }^{1,2}$
}

Received: 19 July 2019 / Revised: 7 October 2019 / Accepted: 22 October 2019 / Published online: 25 November 2019

(c) The Author(s), under exclusive licence to International Spinal Cord Society 2019

\begin{abstract}
Study design Clinical trial.

Objective Spinal cord injury (SCI) impacts autonomic function and bowel management. Bowel care is a potential trigger for autonomic dysreflexia (AD; paroxysmal hypertension elicited by sensory stimuli below the level of lesion). AD can be life threatening so strategies to minimise AD are prioritised after SCI. Lidocaine lubricant is recommended during bowel care with the rationale to minimise the sensory stimulus, reducing AD. The objective of this study was to assess whether lidocaine lubricant (Xylocaine 2\%) ameliorates $\mathrm{AD}$ during at-home bowel care compared with standard lubricant (placebo).

Setting Community.

Method Participants ( $n=13$; age $44.0 \pm 3.3$ years) with high-level SCI (C3-T4) performed their normal at-home bowel care on two days, each time using a different lubricant, with continuous non-invasive cardiovascular monitoring. Injury to spinal autonomic (sympathetic) nerves was determined from low-frequency systolic arterial pressure (LF SAP) variability.

Results Participants displayed reduced autonomic function (LF SAP $3.02 \pm 0.84 \mathrm{mmHg}^{2}$ ), suggesting impaired autonomic control. Bowel care duration was increased with lidocaine $(79.1 \pm 10.0 \mathrm{~min})$ compared to placebo $(57.7 \pm 6.3 \mathrm{~min} ; p=0.018)$. All participants experienced AD on both days, but maximum SAP was higher with lidocaine $(214.3 \pm 10.5 \mathrm{mmHg})$ than placebo $(196.7 \pm 10.0 \mathrm{mmHg} ; p=0.046)$. Overall, SAP was higher for longer with lidocaine $\left(6.5 \times 10^{5} \pm 0.9 \times 10^{5} \mathrm{mmHg} \bullet\right.$ beat) than placebo $\left(4.4 \times 10^{5} \pm 0.6 \times 10^{5} \mathrm{mmHg} \bullet\right.$ beat; $\left.p=0.018\right)$ indicating a higher burden of AD. Heart rate and rhythm disturbances were increased during $\mathrm{AD}$, particularly with lidocaine use.

Conclusions At-home bowel care was a potent trigger for AD. Our findings contradict recommendations for lidocaine use during bowel care, suggesting that anaesthetic lubricants impair reflex bowel emptying, resulting in longer care routines with an increased burden of $\mathrm{AD}$.
\end{abstract}

Victoria E. Claydon

victoria_claydon@sfu.ca

1 Department of Biomedical Physiology and Kinesiology, Simon Fraser University, Burnaby, BC, Canada

2 International Collaboration on Repair and Discoveries (ICORD), University of British Columbia, Vancouver, BC, Canada

3 GF Strong Rehabilitation Centre, Spinal Cord Injury Program, Vancouver Coastal Health, Vancouver, BC, Canada

4 Division of Physical Medicine and Rehabilitation, Faculty of Medicine, UBC, Kelowna, BC, Canada

\section{Introduction}

In addition to the well-known loss of motor and sensory function following traumatic spinal cord injury (SCI), autonomic function is also severely impaired [1-3]. This loss of autonomic function occurs independently of motor and sensory disturbances, and impacts a range of physiological processes. In particular, autonomic dysfunction adversely impacts cardiovascular control and bowel function for individuals living with SCI, with the former complicating the latter [4]. We recently reported that bowel care concerns profoundly impact quality of life for communitydwelling individuals living with SCI, who identified bowel 
management as a key modifiable factor with the potential to improve their quality of life [5].

Bowel impairments after SCI are primarily related to the impact of injury on gastrointestinal function, as the coordination of gastrointestinal motility is dependent on central motor and autonomic control, as well as intrinsic regulation by the enteric nervous system [6]. This neurogenic bowel dysfunction (NBD) is highly dependent on the level of injury. Accordingly, NBD is classified as upper motor neuron (UMN) dysfunction or lower motor neuron (LMN) dysfunction, depending on whether the injury level is above or below the conus medullaris (L1), respectively [6]. UMN lesions maintain reflex activity in the sacral segments of the cord, resulting in loss of voluntary bowel control, with preservation of reflexive defecation. This often results in a spastic sphincter, with constipation and faecal retention that require intervention to trigger defecation [7] - a hyperreflexive bowel. LMN lesions also result in loss of voluntary control of the bowel, but are accompanied by loss of sacral reflexive control with a lax external sphincter, resulting in a high frequency of faecal incontinence-an areflexive bowel.

Individuals with high lesion levels (at or above T6) also commonly experience injury to the autonomic sympathetic nerves responsible for heart rate and blood pressure control. This profound cardiovascular impairment is a key concern for individuals living with SCI and is linked to high cardiovascular morbidity and mortality following SCI [8, 9]. In individuals with high lesion levels, bowel care routines can exacerbate cardiovascular dysfunction by eliciting a condition known as autonomic dysreflexia (AD) [10]. AD is characterized by paroxysmal hypertension in response to sensory stimuli (noxious or non-noxious) below the level of injury [11] that elicits a sympathetic reflex, resulting in widespread vasoconstriction of the splanchnic vascular bed and other vessels below the level of injury. This leads to a dangerous increase in blood pressure, which typically also presents with a marked baroreflex-mediated reduction in heart rate [2, 11-13], and is sustained until the sensory stimulus is removed. Typical causes include a distended bladder or bowel, bladder and bowel care routines, pressure sores, and infections [11, 12]. AD can present clinically with symptoms including profound headache, facial flushing, sweating above the level of injury, blurred vision, increased spasticity, and nausea [11, 12]. The high sympathetic discharge associated with $\mathrm{AD}$ is also proarrhythmogenic, and many individuals report palpitations during AD [14]. However, AD can also present asymptomatically, which is challenging because in the absence of symptoms individuals may not take action to remove the triggering stimulus and resolve the episode [15, 16]. This is important because $\mathrm{AD}$ has been linked to cognitive impairments, cerebral haemorrhage, stroke, myocardial infarction and arrhythmia, and death [9, 17-19].
Bowel care practices [20] (treatment plans designed to minimize or eliminate the occurrence of unplanned or difficult evacuations) are diverse and complex, with individuals often needing a multifactorial approach to bowel management. Bowel care routines [20] (the process of assisted defecation) often require chemical (suppository) and/or mechanical (digital stimulation, manual evacuation, and/or abdominal massage) interventions to elicit reflexive defecation. These methods often employ the use of a lubricant to minimise the potential for damage to the rectal mucosa. We have shown that cardiovascular complications associated with bowel care negatively impact quality of life and are associated with high levels of isolation [5]. Accordingly, AD management should be a target for bowel care intervention $[5,8]$.

One proposed method for reducing $\mathrm{AD}$ severity during bowel care is administration of an anaesthetic lubricant to the rectum, with the goal of mitigating the afferent stimulus, and so blunting the AD response to bowel care [12, 21]. The local anaesthetic lidocaine (a sodium channel blocker) has been recommended for the reduction of $\mathrm{AD}$ severity during bowel care, and is often advocated in clinical bowel care guidelines [11, 12, 15, 21]. However, current clinical recommendations for its use are dated [21] and the evidence regarding the use of lidocaine during bowel care procedures is conflicting [22-24]. We aimed to determine whether the use of a topical anesthetic lubricant (2\% lidocaine) ameliorates cardiovascular complications compared to a placebo lubricant during at-home bowel care. Additional aims include evaluation of the impact of lidocaine on time to complete bowel care, and self-reported symptoms of $\mathrm{AD}$ during care.

\section{Materials and methods}

\section{Participants}

Participants were recruited using a multi-method approach. Print advertisements were circulated through our community partner, Spinal Cord Injury British Columbia, via their quarterly publication, The Spin, and online advertisements were posted through our institutional website (www.icord.org).

Eligible participants were individuals aged $>18$ years of age with chronic ( $>1$ year) traumatic high level (T6 or above) SCI, who had an established bowel care routine, and a prior history of AD. Individuals were excluded if they had a medical/psychiatric condition or substance abuse disorder, used a ventilator, had a colostomy, or did not perform regular bowel care for any reason. Additional exclusion criteria included skin breakdown in the areas receiving pressure during the bowel program, inability to communicate in English, use of medicines containing lidocaine, allergy to lidocaine, and pregnancy. 
Our primary outcome measure was the difference in systolic arterial pressure between the placebo and lidocaine conditions. We determined a meaningful difference to detect in blood pressure between the two study arms of $10 \mathrm{mmHg}$ (based on previous unpublished observations from our lab). With a power of 0.8 and alpha of 0.05 , we determined a sample size of 13 individuals would be necessary to ensure statistical confidence in our ability to detect differences in this primary outcome measure.

\section{Experimental procedure}

We conducted a registered double-blind placebo-controlled crossover clinical trial where participants were randomised to a series of two treatments: lidocaine lubricant (Xylocaine $2 \%$ ) and standard lubricant (KY Jelly; placebo) on two consecutive at-home visits (Fig. 1). The sequence of conditions was determined by random draw, using a complete randomised design, by a researcher external to the research team; seven participants were randomised to the lidocaine arm of the study first, and six to the placebo arm of the study first. Testing took place between August 2016 and October 2018.

Prior to the first visit, participants completed a questionnaire about their bowel management and general bowel continence [5]. On each visit, participants were fitted with a standard three-lead electrocardiogram (ECG; lead II) and a non-invasive beat-to-beat finger blood pressure monitor (Finometer Midi, Finapres Medical Systems [FMS], Amsterdam, Netherlands). After a 10-min baseline recording (sampling rate $200 \mathrm{~Hz}$ ) participants inserted $5 \mathrm{~mL}$ of lubricant into the rectum using a specialised device (Lube Launcher XL, CleanStream, Huntington Beach, USA) to ensure that a minimum amount of lubricant was administered. Following insertion of the lubricant, participants waited 5-min before starting bowel care (to allow for mucosal absorption of lidocaine, which has a typical onset of action of 3-5 min). Participants were then provided with an additional $15 \mathrm{~mL}$ of lubricant and asked to perform their bowel care routine as usual, replacing their normal lubricant with the lubricant provided for that day. The maximum recommended dosage of lidocaine is $600 \mathrm{mg} / 12 \mathrm{~h}$. The dosage provided ( $20 \mathrm{ml}$ of $2 \%$ lidocaine) is equivalent to $400 \mathrm{mg}$. The $20 \mathrm{ml}$ volume was determined based on discussion with bowel care providers and individuals with SCI concerning their typical lubricant needs. This dosing strategy is in line with other similar studies. Given the typical half-life of lidocaine (1.5-2 h) we are confident there was optimal dosing throughout the duration of bowel care.

Five minutes after insertion of the test lubricant, a timer was started and the participant commenced their usual care routine. Bowel care routines were not standardised because the primary interest was to investigate the real-world feasibility and impact of this clinical recommendation in a community setting with usual participant care routines. When the participant signalled the end of their care routine (defined as the time when bowel evacuation was completed, prior to cleaning), the timer was stopped, and the bowel care duration noted. The cardiovascular monitoring equipment was then removed. Participants then completed a questionnaire specific to their bowel care on that day, reporting the method of bowel care employed as well as the severity of cardiovascular symptoms experienced [5]. Self-reported symptoms of AD were determined as described previously [5]. Two participants chose not to complete the post-bowel care questionnaire.

\section{Autonomic dysfunction}

Severity of autonomic dysfunction was determined through spectral analyses of low-frequency systolic arterial pressure variability (LF SAP). LF SAP oscillations $(\sim 0.1 \mathrm{~Hz})$ reflect

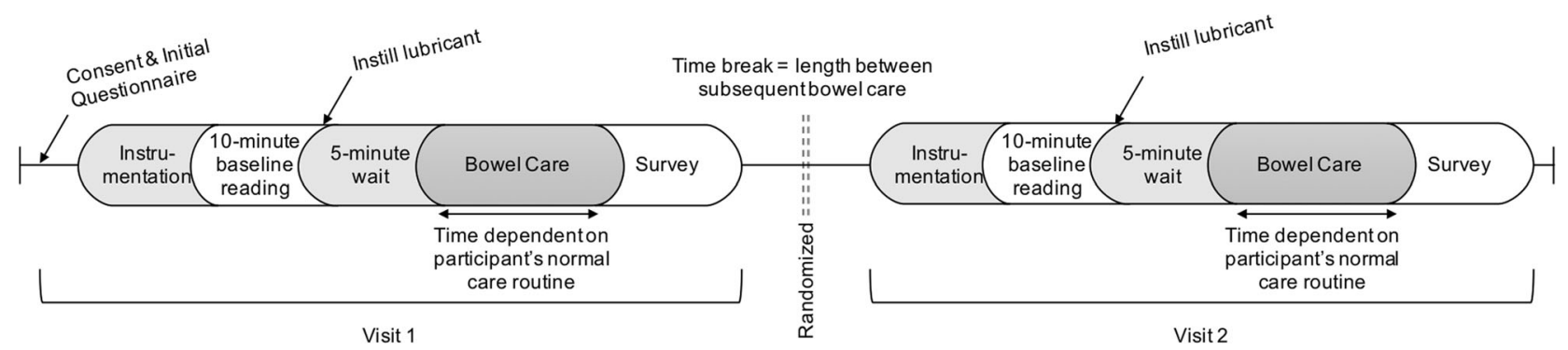

Fig. 1 Protocol schematic. This double-blind placebo-controlled crossover clinical trial took take place over two consecutive visits. Each participant was randomly assigned to a sequence of two treatments: lidocaine lubricant (Xylocaine 2\%) and standard lubricant (KY Jelly; placebo). Participants performed their normal bowel care during two in-home visits, each time with the lubricant assigned for that test day, with continuous non-invasive beat-to-beat cardiovascular monitoring (Finapres Medical Systems (FMS), Amsterdam, Netherlands).
An initial 10-min baseline reading was performed in the position in which participants conducted their bowel care. Participants were then asked to instill $5 \mathrm{~mL}$ of the lubricant using a specialized device (Cleanstream XL Lubricant Launcher, CleanStream) 5-min prior to beginning their care routine. Prior to the first visit, participants were asked to complete a questionnaire about their bowel management and general bowel continence. At the end of each visit, participants completed a second questionnaire specific to their bowel care that day 
sympathetic control of the vasculature, and thus indicate the presence or absence of autonomic cardiovascular control following SCI [25]. Participants with LF SAP lower than $3.75 \mathrm{mmHg}^{2}$ were determined to have autonomicallycomplete injuries.

\section{Cardiac rate and rhythm analyses}

Electrocardiogram data were visually inspected offline after completion of the study. The number of beats that met criteria for bradycardia $(<60 \mathrm{bpm})$ and tachycardia (>100 bpm) was determined during baseline and bowel care, and expressed as a percentage of the total number of beats in the corresponding phase. In addition, each beat was classified as either sinus rhythm, or not, and any arrhythmia or conduction abnormalities noted.

\section{Data analyses}

Beat-to-beat systolic (SAP) and diastolic (DAP) arterial pressures were extracted throughout the testing period. Mean arterial pressure (MAP) was calculated as DAP + $1 / 3 \times($ SAP-DAP $)$. Heart rate was determined from the R-R interval of the ECG. Stroke volume (SV) and cardiac output (Q) were determined using the Modelflow [26, 27], and total peripheral resistance (TPR) was calculated as MAP/Q. Data were collected at a sampling frequency of $200 \mathrm{~Hz}$ and averaged over five successive beats. All parameters were extracted for the entire duration of baseline and bowel care, as well as for only the first 25 min of bowel care. Maximal responses were also determined during bowel care regardless of the time at which they occurred ( Parameter $\left._{\max }\right)$. AD was defined as an increase in $\mathrm{SAP}_{\max }$ of at least $20 \mathrm{mmHg}$ [28] compared with baseline. The overall burden of $\mathrm{AD}$ was determined from the area under the SAP curve (the product of SAP and heart beat).

\section{Statistical analyses}

Data processing was performed using $\mathrm{R}$ (Version 3.3.3) and RStudio (Version 1.1.453). Statistical analyses were performed using Sigmaplot 14 (Systat Software Inc., San Jose, CA). Data were tested for normality and parametric or non-parametric assumptions were used as appropriate. Comparisons of cardiovascular outcomes and symptoms between conditions (lidocaine and placebo) and test phases (baseline and during bowel care) were performed using twoway repeated measures ANOVA. Spearman rank correlations and linear regressions were used to assess relationships between variables. Fisher's exact test was used to evaluate differences in proportions of responses between test conditions. Student $t$ tests were used to compare responses between placebo and lidocaine conditions (e.g. time to complete bowel care). Statistical significance was assumed at $p<0.05$. Where appropriate, data are represented as mean \pm standard error, unless otherwise stated. Researchers, participants, care providers, and those analysing the data were blinded to the test condition. Researchers were unblinded just prior to statistical analyses.

\section{Results}

\section{Demographic and injury characteristics}

Demographic and injury information can be found in Table 1. All participants had high level lesions placing them at risk for cardiovascular complications and $\mathrm{AD}$; therefore, all had UMN NBD with hyperreflexive bowels. All participants had experienced prior documented AD with low resting LF SAP, consistent with severe injury to descending autonomic (sympathetic) pathways.

\section{General bowel practices}

Participant care routines were conducted in a supine sidelying position $(n=6 ; 46 \%)$, or seated on a commode or toilet $(n=7 ; 54 \%)$. Of those who commenced their care routine in a side-lying position (for suppository insertion), five individuals then transferred to a commode or toilet in preparation for and during the passage of stool. Bowel care procedures employed included: rectal suppository $(n=12$; $92 \%)$; digital stimulation $(n=8 ; 62 \%)$; and manual removal of stool $(n=1 ; 8 \%)$, either alone or in combination. Bowel care was performed independently $(n=2 ; 15 \%)$, or with the partial $(n=4 ; 31 \%)$ or total $(n=7 ; 54 \%)$ assistance of a care aide or family member. Bowel care was typically conducted once per day $(n=3 ; 23 \%)$, every other day $(n=$ $9 ; 69 \%)$, and every third day $(n=1 ; 8 \%)$. Each individual employed the same care routine (their usual care) in the same position for both testing days. The mean between visit duration for the two test conditions was $2.5 \pm 0.5$ days.

Table 1 Participant demographic and injury information

Demographic and injury information

Sample size $(n)$

$13(9 \mathrm{M} / 4 \mathrm{~F})$

Age (years)

$44.0 \pm 3.3$

Time post-injury (years)

$13.9 \pm 2.4$

Injury level

C3-T4

Injury severity

AIS A-C

LF SAP $\left(\mathrm{mmHg}^{2}\right)$

$3.02 \pm 0.84$

No. of days between testing

$2.5 \pm 0.5$

Where applicable, data are expressed as mean \pm standard error $L F S A P$ low frequency power of systolic arterial pressure 
One participant was unable to pass stool on the lidocaine test day.

A representative example tracing showing the responses and timing of bowel care in one individual is shown in Fig. 2. AD was triggered by numerous aspects of bowel care, including insertion of lubricant, insertion of suppository, rectal checks, and passage of stool and cleaning.

The time taken to complete bowel care was significantly longer on the lidocaine than the placebo test day (Fig. 3a).

\section{Cardiovascular responses to bowel care}

Baseline cardiovascular parameters were not significantly different between lidocaine and placebo test days (Table 2). Cardiovascular responses during bowel care are shown in Table 2. All participants had an increase in SAP greater than $20 \mathrm{mmHg}$ on each test day, confirming the presence of $\mathrm{AD}$ in both placebo and lidocaine conditions. The magnitude of the blood pressure rise was negatively correlated with the baseline blood pressure $(r=-0.406 ; p=0.04)$. Compared with baseline, the $\Delta \mathrm{SAP}_{\max }$ during placebo was $+80 \pm 8.7 \mathrm{mmHg}$ and during lidocaine was $+90.5 \pm$ $13.4 \mathrm{mmHg}(p=0.386)$; only three individuals had a smaller (reduced by $\geq 10 \mathrm{mmHg}$ ) $\Delta \mathrm{SAP}_{\max }$ with lidocaine than with placebo. $\mathrm{SAP}_{\max }$ during bowel care was significantly higher with lidocaine than placebo (Fig. 3b). The overall burden of $\mathrm{AD}$ on the cardiovascular system (the area under the SAP curve during bowel care) was significantly greater with lidocaine than placebo (Fig. 3c). There was a significant correlation between $\mathrm{SAP}_{\max }$ during bowel care and the overall burden of AD (Fig. 4). On both test days, participants also experienced profound bradycardia with large and significant increases in $\mathrm{SV}_{\max }, \mathrm{Q}_{\max }$, and $\mathrm{TPR}_{\max }$ during bowel care compared with baseline, and compared with the mean values during the whole period of bowel care (Table 2). $\mathrm{SV}_{\max }$ was smaller on the lidocaine than the placebo test day $(p=0.005)$.

We considered the possibility that the lidocaine was effective at reducing the severity of $\mathrm{AD}$, but only during its presumed maximum efficacy, within the first $25 \mathrm{~min}$ after insertion of the lubricant. Accordingly, we compared the $\mathrm{SAP}_{\max }$ (lidocaine: $172.9 \pm 8.4 \mathrm{mmHg}$; placebo: $182.8 \pm$ $10.3 \mathrm{mmHg} ; p=0.313$ ) and AD burden, expressed as the area under the SAP curve (lidocaine: $1.8 \times 10^{5} \pm 0.1 \times 10^{5}$ $\mathrm{mmHg}$; placebo: $1.8 \times 10^{5} \pm 0.2 \times 10^{5} \mathrm{mmHg} ; \quad p=0.9$ ), between the two conditions over the initial $25 \mathrm{~min}$ of bowel care. Again we found no significant benefit of lidocaine use for the amelioration of $\mathrm{AD}$.

\section{ECG responses to bowel care}

Rate changes during sinus rhythm The incidence of abnormal heart rates was higher during bowel care

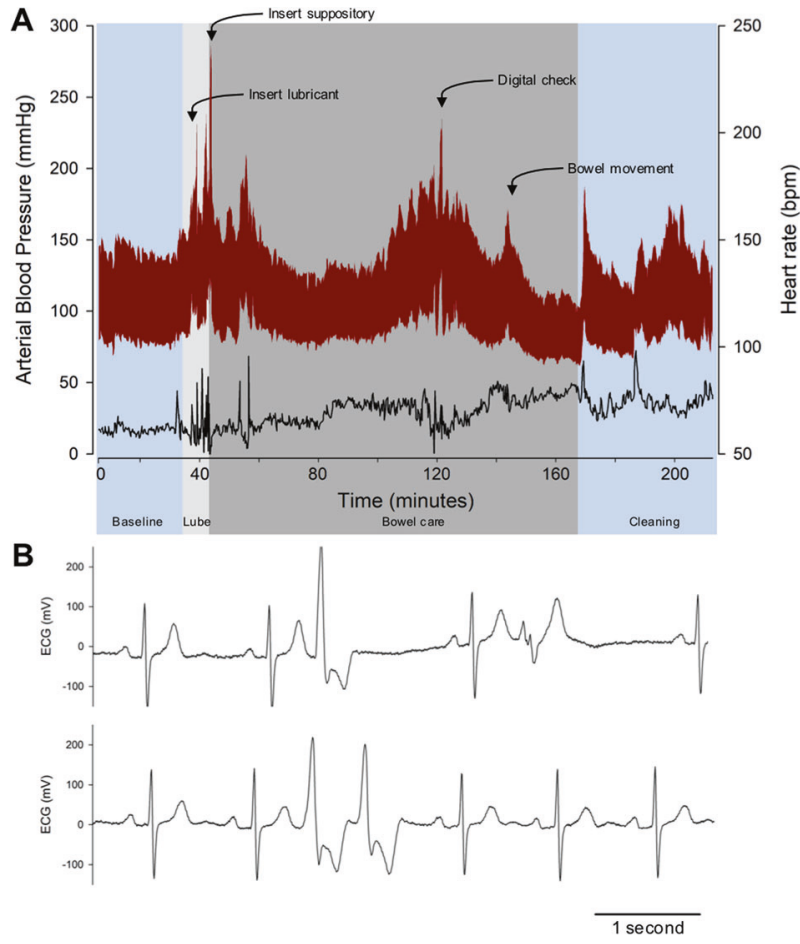

Fig. 2 Representative example tracing showing arterial blood pressure, heart rate, and ECG responses to bowel care. a Beat-to-beat blood pressure and heart rate recording from an individual with a C4 AIS A injury collected on the lidocaine test day. Resting blood pressure (red) was slightly elevated, perhaps reflecting mild colorectal distension induced autonomic dysreflexia (AD) prior to beginning care. Episodic severe $\mathrm{AD}$ was provoked by bowel care procedures including insertion of lubricant and suppository, as well as rectal checks and the passage of stool. Note, cleaning also induced a severe AD response. Concurrent heart rate changes (black) during $\mathrm{AD}$ episodes reflect the presence of arrhythmia or abnormal heart rate control during this severe sympathetic stimulus. Interestingly, AD often triggered paradoxical tachycardia despite providing a strong stimulus for baroreflex mediated bradycardia. The grey shaded region indicates the duration of bowel care. b Representative example ECG traces showing cardiac abnormalities in an individual with a C5 AIS A injury provoked during episodes of AD induced by bowel care, collected on the lidocaine test day. Top; multifocal ventricular ectopics occurring in bigeminy. Bottom; couplet of ventricular ectopics. $E C G$ electrocardiogram

compared with baseline for bradycardia $(p=0.036)$ and tended to be higher for tachycardia $(p=0.07)$ independent of the placebo or lidocaine condition (Fig. 5). There were no significant differences between placebo and lidocaine conditions in the incidence of episodes of bradycardia or tachycardia at baseline or during bowel care.

Rhythm and conduction abnormalities Cardiac arrhythmia were common during bowel care (Fig. 2). Considering all causes of arrhythmia independent of location of origin in the cardiac conducting system, there were more events during bowel care compared with baseline in the lidocaine condition $(p=0.057)$, but not in the placebo condition ( $p=$ 0.293). The number of all-cause arrhythmic events during bowel care was greater in lidocaine than placebo $(p=$ 
A

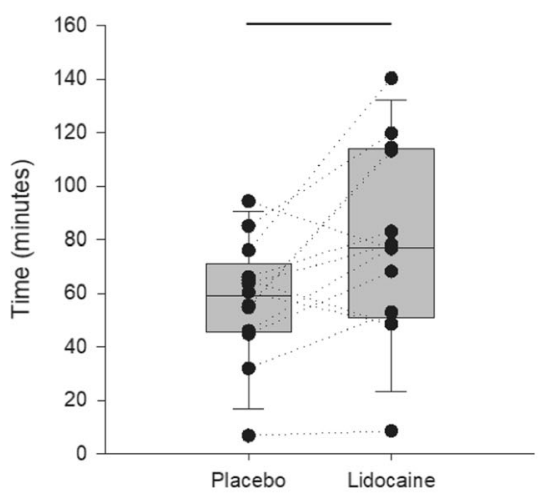

B

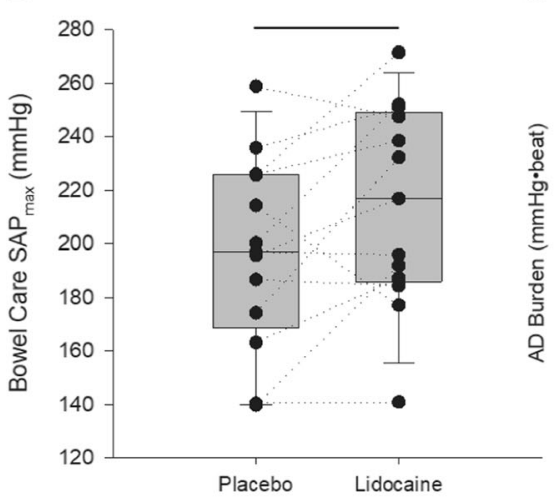

C

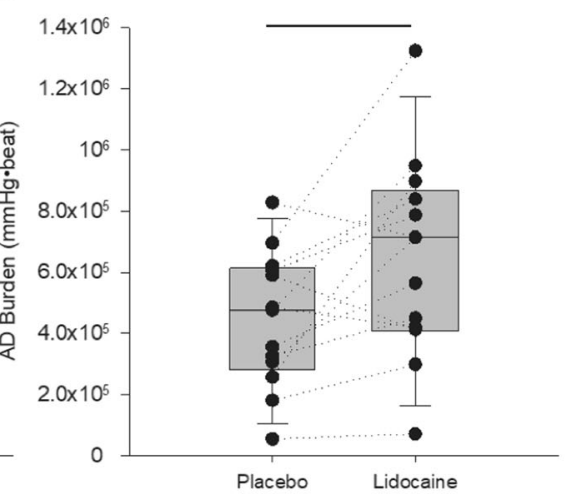

Fig. 3 Lidocaine use was associated with adverse bowel care outcomes. a Lidocaine prolonged the time to complete bowel care when compared with the placebo condition $(p=0.018)$. b Lidocaine use was associated with more severe autonomic dysreflexia (AD), with a higher maximum systolic arterial blood pressure $\left(\mathrm{SAP}_{\max }\right)$ during bowel care in the lidocaine than placebo condition $(p=0.046)$. $\mathbf{c}$ Lidocaine use increased the burden of $\mathrm{AD}$ compared with placebo $(p=0.018)$, defined as the area under the systolic arterial pressure curve during bowel care (the product of systolic arterial pressure and heart beat). $S A P$ systolic arterial pressure
Table 2 Responses to bowel care in placebo and lidocaine conditions

\begin{tabular}{|c|c|c|c|}
\hline & Placebo & Lidocaine & Between group differences \\
\hline $\begin{array}{l}\text { Time to complete bowel } \\
\text { care (min) }\end{array}$ & $57.7 \pm 6.3$ & $79.1 \pm 10.0^{\mathrm{c}}$ & $21.5 \pm 7.8[6.2-36.7]$ \\
\hline \multicolumn{4}{|l|}{ Baseline } \\
\hline RRI (ms) & $892 \pm 42$ & $896 \pm 48$ & $4.4 \pm 20.2[-35.2-44.0]$ \\
\hline SAP (mmHg) & $116.7 \pm 5.0$ & $123.8 \pm 7.2$ & $7.1 \pm 5.2[-3.0-17.3]$ \\
\hline $\mathrm{SV}(\mathrm{mL})$ & $79.3 \pm 5.6$ & $81.5 \pm 6.9$ & $2.1 \pm 6.7[-11.0-15.3]$ \\
\hline $\mathrm{Q}(\mathrm{L} / \mathrm{min})$ & $5.42 \pm 0.3$ & $5.4 \pm 0.4$ & $0.1 \pm 0.4[-0.7-0.9]$ \\
\hline $\mathrm{TPR}(\mathrm{mmHg} \bullet \mathrm{min} / \mathrm{L})$ & $18.0 \pm 1.4$ & $18.3 \pm 1.8$ & $0.3 \pm 2.1[-3.8-4.3]$ \\
\hline \multicolumn{4}{|l|}{ Bowel care } \\
\hline $\mathrm{RRI}_{\text {mean }}(\mathrm{ms})$ & $908 \pm 47$ & $910 \pm 50$ & $1.7 \pm 19.0[-35.5-39.0]$ \\
\hline $\mathrm{SAP}_{\text {mean }}(\mathrm{mmHg})$ & $126.7 \pm 7.5$ & $135.7 \pm 6.7$ & $9.0 \pm 5.3[-1.4-19.4]$ \\
\hline $\mathrm{SV}_{\text {mean }}(\mathrm{mL})$ & $77.2 \pm 7.2$ & $70.6 \pm 7.2$ & $-6.6 \pm 3.5[-13.4-0.2]$ \\
\hline $\mathrm{Q}_{\text {mean }}(\mathrm{L} / \mathrm{min})$ & $5.24 \pm 0.4$ & $4.79 \pm 0.4$ & $-0.5 \pm 0.2[-0.9-0.0]$ \\
\hline $\mathrm{TPR}_{\text {mean }}(\mathrm{mmHg} \bullet \min / \mathrm{L})$ & $21.4 \pm 1.7$ & $26.3 \pm 3.4$ & $4.9 \pm 2.7[-0.4-10.1]$ \\
\hline $\mathrm{RRI}_{\max }(\mathrm{ms})$ & $2038 \pm 359^{\mathrm{ab}}$ & $1808 \pm 182^{\mathrm{ab}}$ & $-230 \pm 410[-1033-574]$ \\
\hline $\mathrm{SAP}_{\max }(\mathrm{mmHg})$ & $196.7 \pm 10.0^{\mathrm{ab}}$ & $214.3 \pm 10.5^{\mathrm{abc}}$ & $17.6 \pm 7.9[2.1-33.1]$ \\
\hline $\mathrm{SV}_{\max }(\mathrm{mL})$ & $147.5 \pm 12.3^{\mathrm{ab}}$ & $120.8 \pm 8.9^{\mathrm{abc}}$ & $-41.7 \pm 19.1[-79.2-4.3]^{\mathrm{a}}$ \\
\hline $\mathrm{Q}_{\max }(\mathrm{L} / \mathrm{min})$ & $11.18 \pm 1.4^{\mathrm{ab}}$ & $9.36 \pm 0.76^{\mathrm{ab}}$ & $22.9 \pm 42.2[-59.8-105.7]$ \\
\hline $\mathrm{TPR}_{\max }(\mathrm{mmHg} \cdot \min / \mathrm{L})$ & $111.1 \pm 20.9^{\mathrm{ab}}$ & $94.63 \pm 13.1^{\mathrm{ab}}$ & $-46.2 \pm 39.2[-123.0-30.7]$ \\
\hline $\mathrm{AuC}(\mathrm{mmHg} \bullet$ beats $)$ & $\begin{array}{l}4.4 \times 10^{5} \pm \\
0.6 \times 10^{5}\end{array}$ & $6.5 \times 10^{5} \pm 0.9 \times 10^{5 c}$ & $\begin{array}{l}2.0 \times 10^{5} \pm 0.7 \times 10^{5}[0.6 \times \\
\left.10^{5}-3.5 \times 10^{5}\right]\end{array}$ \\
\hline$\Delta \mathrm{SAP}_{\max }(\mathrm{mmHg})$ & $+80 \pm 8.7$ & $+90.5 \pm 13.4$ & $10.5 \pm 11.7[-12.4-33.4]$ \\
\hline
\end{tabular}

Data are expressed as mean \pm standard error. Differences between conditions (lidocaine-placebo) are expressed as mean \pm standard error [confidence interval]

$R R I$ RR interval, $S A P$ systolic arterial pressure, $S V$ stroke volume, $Q$ cardiac output, $T P R$ total peripheral resistance

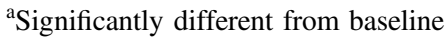

${ }^{\mathrm{b}}$ Significantly different from bowel care mean

${ }^{\mathrm{c}}$ Significantly different from placebo condition 


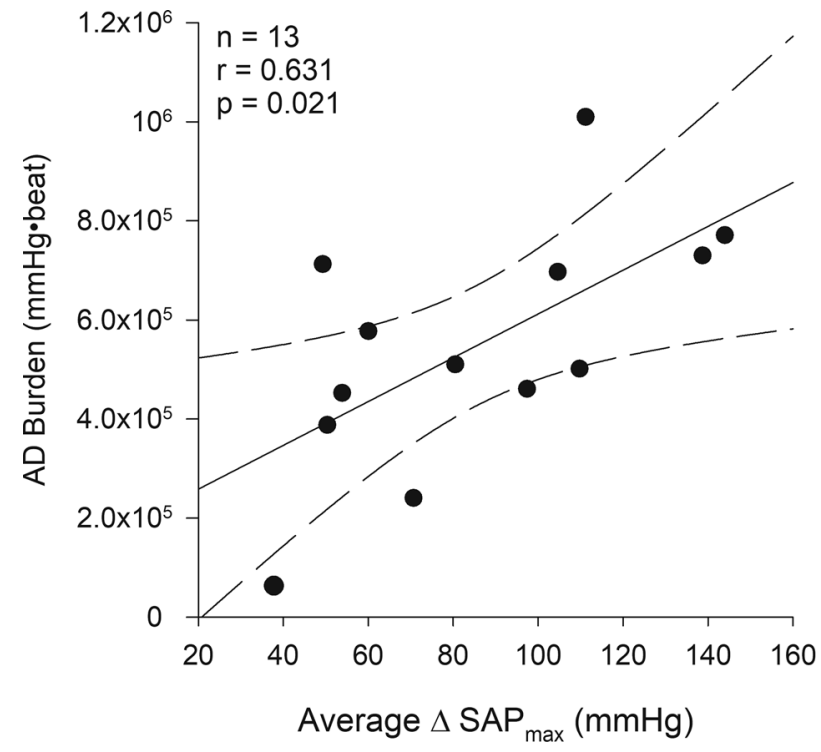

Fig. 4 Association between maximum systolic arterial pressure response to bowel care and the overall burden of autonomic dysreflexia. Larger systolic arterial pressure responses, expressed as the maximum change from baseline $\left(\Delta \mathrm{SAP}_{\max }\right)$ were associated with a greater burden of autonomic dysreflexia (defined as the area under the systolic arterial pressure curve during bowel care, the product of systolic arterial pressure and heart beat). Responses were averaged for each participant for the lidocaine and placebo conditions. SAP systolic arterial pressure

0.011). In the lidocaine condition, but not during placebo, there were significantly more arrhythmic events originating from the sinus node, atria, and ventricles, but not the atrioventricular node, during bowel care compared with baseline. During bowel care there tended to be more events occurring in the sinus node $(p=0.052)$, atria $(p=0.084)$, and ventricles $(p=0.051)$ in the lidocaine than placebo condition.

\section{Participant perspectives}

Symptoms typical of AD were reported by $100 \%$ of individuals during the lidocaine condition and $90 \%$ of individuals during the placebo condition- $80 \%$ of individuals thought they had experienced AD on the lidocaine and $90 \%$ on the placebo test day. The mean AD symptom score was the same in the lidocaine $(7.6 \pm 1.5)$ and placebo conditions $(7.0 \pm 2.0, p=0.8)$. The AD symptom score was not significantly correlated with the severity of AD observed when quantified as either $\mathrm{SAP}_{\max }(r=0.254, p=0.27)$ or the $\mathrm{AD}$ burden $(r=0.191, p=0.4)$.

Symptoms of palpitations were reported by $3 / 11(27 \%)$ individuals during the lidocaine condition and 2/11 (18\%) individuals during the placebo condition. When considering whether they normally experienced symptoms of palpitations during their bowel care 5/13 (38\%) of participants felt that they did (2 rarely; 1 weekly; 2 daily).

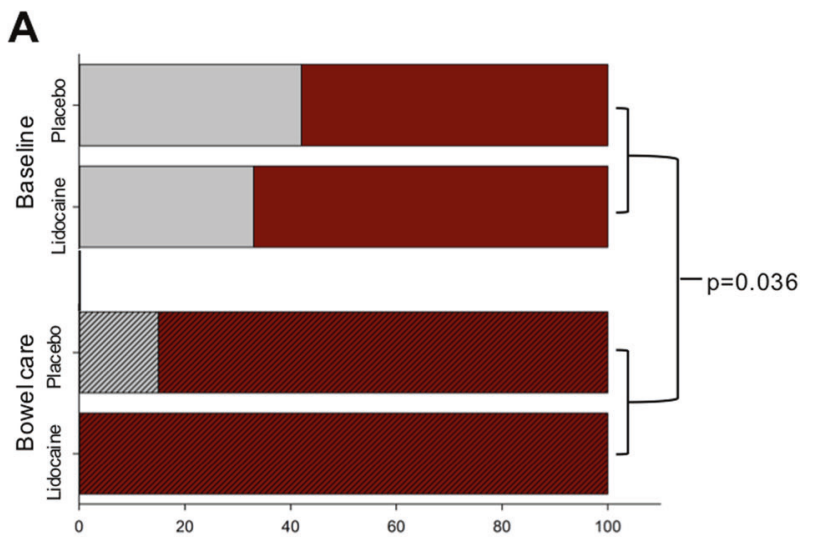

B

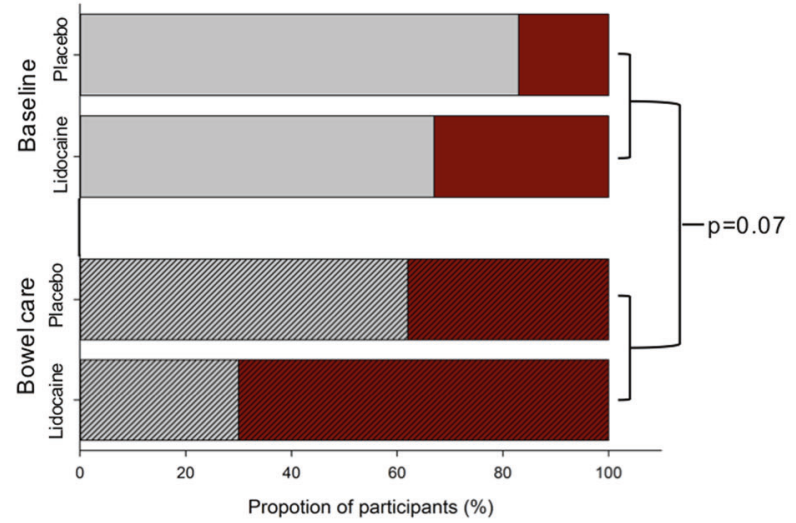

Fig. 5 Proportion of abnormal heart rates at baseline and during bowel care. Incidence of bradycardia ( $a<60$ beats per minute) and tachycardia (b >100 beats per minute) during baseline and bowel care in both placebo and lidocaine conditions. Grey boxes indicate the absence of abnormal beats while red boxes indicate the presence of abnormal beats. There was a significant main of test phase, whereby a higher proportion of individuals experienced episodes of bradycardia $(p=0.036)$ and tachycardia $(p=0.07)$ during bowel care compared with baseline for the group. There were no significant differences between placebo and lidocaine conditions in the incidence of episodes of bradycardia or tachycardia, either at baseline or during bowel care

Participants were asked about their perceptions of their bowel care on each test day. They reported that it was worse on the lidocaine day compared with the placebo test day, commonly reporting that initiation of bowel movements was prolonged with lidocaine, with time to empty being longer and more difficult compared with their normal care.

\section{Discussion}

This is the first study to continuously monitor cardiovascular responses during at-home bowel care in individuals with SCI. We showed that, despite the heterogeneity of both SCI and care routines, it is possible to make reliable and accurate cardiovascular assessments during personal care routines. 
Our main findings were contrary to our hypothesis-we demonstrated that the use of lidocaine lubricant actually worsened the severity of $\mathrm{AD}$ during at-home bowel care. This was associated with an increased time to complete care using lidocaine. This may reflect an inadvertent effect of afferent blockade on reflexive bowel emptying-necessitating longer bowel care routines with greater stimuli required to trigger emptying and so exacerbating $\mathrm{AD}$. For example, digital stimulation techniques and use of suppositories rely on intact nociceptive and proprioceptive spinal reflexes. It may be that lidocaine use impairs these reflexes and prolongs the duration of bowel care.

We also found that episodes of $\mathrm{AD}$ induced by bowel care provoked abnormal heart rate responses and cardiac arrhythmia, with rate and rhythm disturbances exacerbated during the lidocaine condition, when the $\mathrm{AD}$ was more severe and more prolonged. The increased risk of arrhythmia during $\mathrm{AD}$ has been noted previously [14, 29-31] particularly in individuals with high level autonomicallycomplete lesions [14]. Interestingly, the incidence of reported symptoms of palpitations during typical bowel care in the present study (38\%) was similar to our previous survey of 287 individuals with SCI (32\%) [5]. These data highlight the relationships between $\mathrm{AD}$, heart rate and rhythm abnormalities, and symptoms of palpitations in individuals with SCI, and their strong association with bowel care. The greater susceptibility to heart rate and rhythm disorders during bowel care in the lidocaine condition may reflect the more severe $\mathrm{AD}$ in that condition triggering cardiac abnormalities, and/or a direct effect of circulating lidocaine on cardiac sodium channels.

In terms of the participant perception of the treatment, lidocaine use did not improve symptoms of AD or palpitations, and participant questionnaires revealed that they perceived their care routines to have taken longer and bowel emptying to have been more difficult to complete in the lidocaine condition.

These data are important because currently lidocaine lubrication is recommended during bowel care based on the rationale that it would, in theory, ameliorate $\mathrm{AD}$ [21, 32]. Based on our preliminary data these recommendations are not correct, and novel therapies to blunt $\mathrm{AD}$ without exacerbation of the time taken to undertake bowel care are imperative. Use of lidocaine lubrication during routine bowel care should not be recommended.

Our data suggest that lidocaine use should not be recommended during routine at-home bowel care for the amelioration of $\mathrm{AD}$, and we believe this is important information for the clinical and stakeholder community. However, the inability to use lidocaine to ameliorate AD during bowel care raises the question: what is the best solution to mitigate $\mathrm{AD}$ induced by routine at-home bowel care? Procedures that shorten the bowel care duration might minimise the overall burden of $\mathrm{AD}$, but may not improve the maximum blood pressure or symptoms provoked by bowel care. Hypotensive pharmaceutical agents might blunt blood pressure spikes during $\mathrm{AD}$, but would have a long lasting effect that would place individuals at risk for syncopal events and orthostatic hypotension once the period of $\mathrm{AD}$ is over. There are initial case reports showing promising responses to epidural electrical stimulation to acutely modulate bowel function in motor-complete SCI, decreasing the time to complete bowel care [33, 34]. Reports are conflicting, however, as to whether this stimulation improves bowel dysfunction according to clinical outcomes $[33,34]$. These initial findings underscore the need to replicate these results in larger scale placebo-controlled double-blind crossover clinical trials.

One notable observation was the burden bowel care places on individuals with SCI - all participants experienced severe $\mathrm{AD}$, even in the placebo condition, with SAP $_{\max }$ of approximately $200 \mathrm{mmHg}$. Associated palpitations, abnormal heart rates, cardiac rhythm disturbances, and symptoms of $\mathrm{AD}$ were prevalent. The duration of bowel care was long, almost 1-h on average, even without considering cleaning, dressing, and transfers. These data underscore the negative impact of bowel care on quality of life for individuals with SCI [5, 35, 36]. Despite high levels of dissatisfaction with bowel care, the majority of individuals with SCI are not actively modifying and optimising their care routines [5]. This may reflect a perceived lack of options for improvement in bowel care, with a lack of evidence-based clinical guidelines, and many clinical recommendations (such as the use of lidocaine lubricant to ameliorate $\mathrm{AD}$ ) that, despite being based on sound rationale, do not work as intended. Further research into effective strategies to improve bowel care and associated cardiovascular complications should be prioritised.

This study did not assess the impact of anesthetic administration during anorectal procedures. However, previous research demonstrated that topical rectal lidocaine did not blunt the AD response to anorectal procedures [24]. There is evidence that intersphincteric anal block with $1 \%$ lidocaine injection can blunt $\mathrm{AD}$ responses during anorectal procedures [23]. Our findings may not apply to intersphincteric anal block during anorectal procedures, where efficient bowel emptying is not the target.

The current treatment algorithm for the emergency management of acute hypertensive crises during $\mathrm{AD}$ recommends use of lidocaine lubricant while performing bowel checks and manual removal of any stool present that may be triggering or contributing to the episode [21]. Although we did not examine the impact of lidocaine lubricant on bowel procedures performed as part of the acute management of $\mathrm{AD}$, our data call into question the efficacy of this approach. Based on our results it is possible 
that use of anaesthetic lubricant while assessing bowel triggers for acute episodes of AD would impair the removal of stool, and increase the incidence of heart rate and rhythm disturbances, without blunting the blood pressure rise. More research is needed to confirm the best approach for the management of acute hypertensive crises in individuals with SCI that have a bowel trigger.

It should be noted that baseline readings were taken just prior to the initiation of bowel care. As bowel care was performed according to each participant's normal bowel care schedule, we expect that their bowel was distended during this baseline reading, and that this might have induced mild AD at baseline. Despite this, all participants had blood pressure rises upon the initiation of bowel care that far exceeded the clinical definitions of $\mathrm{AD}$ [28]. Indeed, the rise in $\mathrm{SAP}_{\max }$ may have underestimated the true magnitude of the blood pressure rise during $\mathrm{AD}$ in cases where the baseline SAP was already elevated as a consequence of the presence of faecal material distending the rectum prior to starting bowel care. Interestingly, the $\mathrm{SAP}_{\max }$ response during at-home bowel care far exceeded that of a previous study examining cardiovascular responses to a standardised in-hospital bowel routine performed by nursing staff [22]. In the earlier study blood pressure was taken intermittently rather than beat-to-beat, so it is probable that the maximum blood pressure rise was not captured. It is also noteworthy that this previous study reported some benefit from use of lidocaine during the procedure in terms of the $\mathrm{SAP}_{\text {max }}$ (lidocaine $+33.2 \pm 14.6 \mathrm{mmHg}$; placebo $+50.2 \pm 19.5 \mathrm{mmHg}, p<0.001$ ) [22]. Our data suggest that this does not extend to at-home care, and may not reflect the true severity of AD due to the intermittent blood pressure recordings. Another consideration is that the time to complete bowel care or presence of arrhythmia was not reported in the previous study-even if a modest reduction in SAP were obtained during in-hospital bowel care, if this came at the cost of extended bowel care duration or increased arrhythmia, the potential for benefit from lidocaine use may be outweighed.

We designed this study to evaluate the impact of lidocaine on cardiovascular responses to usual bowel care, rather than a standardized bowel protocol. By focusing on usual care, we were able to ascertain whether lidocaine use is a feasible option for ameliorating $\mathrm{AD}$ in community dwelling individuals - the reality of bowel care for most people living with SCI. We recognise that the incidence/ severity of $\mathrm{AD}$ is related to the method of bowel management [4, 37]. In our study not all individuals used the same management approach; however, participants did employ the same management strategy on both test days, so it is unlikely that variations in the bowel care approach influenced the outcomes of our study. We consider that the use of real-world bowel care enhances the applicability of our findings to the SCI community.
We limited our study to individuals with high-level SCI who were known to have AD and had a consistent bowel care routine. While this means that our results may not extend to all individuals with SCI, they are likely to extend to the target user group - those with lesions placing them at risk for $\mathrm{AD}$ who know that they have experienced it and/or are troubled by symptoms of AD. Interestingly, while our participants were predominantly able to identify the presence of $\mathrm{AD}$, their symptom scores were quite low, and did not reflect the severity of the blood pressure rise. Asymptomatic, or silent $\mathrm{AD}[15,16,37]$, has been documented during other potent triggers for $\mathrm{AD}$, underscoring the disconnect between symptoms of $\mathrm{AD}$ and the severity of the blood pressure rise.

While not the purpose of the study, it is possible that these data provide additional information as to the nature of the triggering stimulus for AD. Given that local anaesthesia was ineffective at reducing $\mathrm{AD}$ during bowel care, this could indicate that deep visceral stimuli, such as peristaltic contractions initiated during the bowel routine, are capable of triggering $\mathrm{AD}$. This mechanistic insight could aid the development of bowel routines and/or treatments that minimize triggers for $\mathrm{AD}$.

\section{Limitations}

We had a relatively small sample size for this study, and of course this has the potential to impact statistical power. We performed our sample size calculation for the primary outcome measure (the change in $\mathrm{SAP}_{\max }$ during bowel care) and this was adequately powered. We conducted interim analyses once our target sample size for our primary outcome measure was reached, and given the clear and statistically robust demonstration of a detrimental effect of the lidocaine on bowel care, AD and the incidence of abnormal heart rates and arrhythmia, we chose not to continue our recruitment beyond that initial target.

In order to conclude that the lidocaine was ineffective, we must be certain that the dosage and timing were appropriate. We are confident that we had optimal dosing based on the known effective dosage, time of onset of action, and half-life of mucosal lidocaine. We also examined our data over the first $25 \mathrm{~min}$ of bowel care in case the impact of the lidocaine administration was beginning to wane in some of the individuals with particularly long care routines-our results were unchanged. Lidocaine was not beneficial.

\section{Conclusions}

Enhancement of bowel care and improvement of associated cardiovascular complications has been highlighted as a 
priority by individuals with SCI. Routine at-home bowel care is a significant trigger for $\mathrm{AD}$ and associated with heart rate and rhythm abnormalities in individuals with high level SCI. Use of lidocaine lubricant with the rationale of blocking the afferent trigger for AD is not effective: it prolongs bowel care, worsens $\mathrm{AD}$ and increases the incidence of cardiac abnormalities. Lidocaine lubricants should not be recommended for routine bowel care in individuals with SCI.

\section{Data availability}

Due to legal and ethical restrictions, data cannot be made publicly available. Data will be made available upon request; however, only aggregated data may be in the public domain according to the stipulations from our research ethics board with respect to the maintenance of confidentiality. Additional published or public analyses would only be permitted with ethics approval for secondary data access, and only with aggregated analyses. Requests can be sent to Dina Shafey, Associate Director, Office of Research Ethics, Simon Fraser University (dshafey@sfu.ca).

Acknowledgements We are grateful to Spinal Cord Injury British Columbia and the International Collaboration On Repair Discoveries for helping to raise awareness of this study.

Funding This work was funded by the Craig H Neilsen Foundation.

Author contributions JAI and VEC conceived the idea of the study. JAI, VEML, and VEC designed the study protocol. VEML and MM collected the data. VEML and SS analysed the data. MM and RW provided clinical oversight. VEML and VEC wrote the manuscript. VEC supervised the research. All authors contributed to the critical revision of the manuscript.

\section{Compliance with ethical standards}

Statement of ethics This study was approved by the Department of Research Ethics at Simon Fraser University and conforms to the principles outlined in the Declaration of Helsinki [38]. We certify that all applicable institutional and governmental regulations concerning the ethical use of human volunteers were followed during the course of this research. Participants provided written informed consent and the trial was registered (Clinical.Tials.gov \#NCT01567605).

Conflict of interest The authors declare that they have no conflict of interest.

Publisher's note Springer Nature remains neutral with regard to jurisdictional claims in published maps and institutional affiliations.

\section{References}

1. Karlsson A-K. Autonomic dysfunction in spinal cord injury: clinical presentation of symptoms and signs. Prog Brain Res. 2006;152:1-8.
2. Anderson K. Autonomic Dysfunction After Spinal Cord Injury. Prog Brain Res. 2006;152:xi-xii.

3. Claydon VE, Steeves JD, Krassioukov AV. Orthostatic hypotension following spinal cord injury: understanding clinical pathophysiology. Spinal Cord. 2006;44:341-51.

4. Stiens SA, Biener Bergman S, Goetz LL. Neurogenic bowel dysfunction after spinal cord injury: clinical evaluation and rehabilitative management. Arch Phys Med Rehabil. 1997;78:S86-102.

5. Inskip JA, Lucci V-EM, McGrath MS, Willms R, Claydon VE. A community perspective on bowel management and quality of life after spinal cord injury: the influence of autonomic dysreflexia. J Neurotrauma. 2018;35:1091-105.

6. Enck P, Greving I, Klosterhalfen S, Wietek B. Upper and lower gastrointestinal motor and sensory dysfunction after human spinal cord injury. Prog Brain Res. 2006;152:373-84.

7. Coggrave M, Mills P, Willms R, Eng JJ. Bowel dysfunction and management following spinal cord injury. In Eng JJ, Teasell RW, Miller WC, Wolfe DL, Townson AF, Hsieh JTC, Connolly SJ, Noonan VK, Loh E, McIntyre A, editors. Spinal Cord Injury Rehabilitation Evidence. Version 5.0. Vancouver: 2014. p. 1-48.

8. Anderson KD. Targeting recovery: priorities of the spinal cordinjured population. J Neurotrauma. 2004;21:1371-83.

9. Cragg JJ, Noonan VK, Krassioukov AV, Borisoff JF. Cardiovascular disease and spinal cord injury: results from a national population health survey. Neurology. 2013;81:723-8.

10. Kursh ED, Freehafer A, Persky L. Complications of autonomic dysreflexia. J Urol. 1977;118:70-2.

11. Blackmer J. Rehabilitation medicine: 1. Autonomic dysreflexia. Can Med Assoc J. 2003;169:931-5.

12. Bycroft J, Shergill IS, Choong EAL, Arya N, Shah JR. Autonomic dysreflexia: a medical emergency. Postgr Med J. 2005;81:232-5.

13. Rabchevsky AG. Segmental organization of spinal reflexes mediating autonomic dysreflexia after spinal cord injury. Prog Brain Res. 2006;152:265-74.

14. Ravensbergen HJC, Walsh ML, Krassioukov AV, Claydon VE. Electrocardiogram-based predictors for arrhythmia after spinal cord injury. Clin Auton Res. 2012;22:265-73.

15. Kirshblum SC, House JG, O'Connor KC. Silent autonomic dysreflexia during a routine bowel program in persons with traumatic spinal cord injury: a preliminary study. Arch Phys Med Rehabil. 2002;83:1774-6.

16. Elliott S, Krassioukov A. Malignant autonomic dysreflexia in spinal cord injured men. Spinal Cord. 2006;44:386-92.

17. Phillips AA, Ainslie PN, Warburton DER, Krassioukov AV. Cerebral blood flow responses to autonomic dysreflexia in humans with spinal cord injury. J Neurotrauma. 2016;33:315-8.

18. Phillips A, Matin N, West C, Zheng A, Galea L, Dorrance A, et al. Autonomic dysreflexia impairs cerebrovascular health and cognition in experimentals spinal cord injury. FASEB J. 2015;29:800.10.

19. Wecht JM, Bauman WA. Decentralized cardiovascular autonomic control and cognitive deficits in persons with spinal cord injury. J Spinal Cord Med. 2013;36:74-81.

20. Clinical practice guidelines: Neurogenic bowel management in adults with spinal cord injury. Spinal Cord Medicine Consortium. J Spinal Cord Med. 1998;21:248-93.

21. Consortium for Spinal Cord Medicine. Acute management of autonomic dysreflexia: individuals with spinal cord injury presenting to health-care facilities. J Spinal Cord Med. 2002;25: Suppl 1:S67-88.

22. Furusawa K, Sugiyama H, Tokuhiro A, Takahashi M, Nakamura T, Tajima F. Topical anesthesia blunts the pressor response induced by bowel manipulation in subjects with cervical spinal cord injury. Spinal Cord. 2009;47:144-8. 
23. Cosman BC, Vu TT. Lidocaine anal block limits autonomic dysreflexia during anorectal procedures in spinal cord injury: a randomized, double-blind, placebo-controlled trial. Dis Colon Rectum. 2005;48:1556-61.

24. Cosman BC, Vu TT, Plowman BK. Topical lidocaine does not limit autonomic dysreflexia during anorectal procedures in spinal cord injury: a prospective, double-blind study. Int J Colorectal Dis. 2002;17:104-8.

25. Claydon VE, Krassioukov AV. Clinical correlates of frequency analyses of cardiovascular control after spinal cord injury. Am J Physiol. 2007;294:H668-78.

26. van Lieshout J, Toska K, van Lieshout E, Eriksen M, Walloe L, Wesseling K. Beat-to-beat noninvasive stroke volume from arterial pressure and Doppler ultrasound. Eur J Appl Physiol. 2003;90:131-7.

27. Dyson KS, Shoemaker JK, Arbeille P, Hughson RL. Modelflow estimates of cardiac output compared with Doppler ultrasound during acute changes in vascular resistance in women. Exp Physiol. 2010;95:561-8.

28. Krassioukov A, Biering-Sørensen F, Donovan W, Kennelly M, Kirshblum S, Krogh K, et al. International standards to document remaining autonomic function after spinal cord injury. J Spinal Cord Med. 2012;35:201-10.

29. Hector SM, Biering-Sørensen T, Krassioukov A, Biering-Sørensen F. Cardiac arrhythmias associated with spinal cord injury. J Spinal Cord Med. 2013;36:591-9.

30. Claydon VE, Elliott SL, Sheel AW, Krassioukov A. Cardiovascular responses to vibrostimulation for sperm retrieval in men with spinal cord injury. J Spinal Cord Med. 2006;29:207-16.
31. Forrest GP. Atrial fibrillation associated with autonomic dysreflexia in patients with tetraplegia. Arch Phys Med Rehabil. 1991;72:592-4.

32. Furusawa K, Sugiyama H, Ikeda A, Tokuhiro A, Koyoshi H, Takahashi M, et al. Autonomic dysreflexia during a bowel program in patients with cervical spinal cord injury. Acta Med Okayama. 2007;61:221-7.

33. Hubscher $\mathrm{CH}$, Herrity AN, Williams CS, Montgomery LR, Willhite AM, Angeli CA, et al. Improvements in bladder, bowel and sexual outcomes following task-specific locomotor training in human spinal cord injury. PLoS One. 2018;13:e0190998.

34. Walter M, Lee X, Kavanagh AH, Phillips A, Krassioukov AA. A V. Epidural spinal cord stimulation acutely modulates lower urinary tract and bowel function following spinal cord injury: a case report. Front phsyiology. 2018;9:1-7.

35. Coggrave M, Norton C, Wilson-Barnett J. Management of neurogenic bowel dysfunction in the community after spinal cord injury: a postal survey in the United Kingdom. Spinal Cord. 2009;47:323-33.

36. Kim J, Koh E, Leigh J, Shin H-I. Management of bowel dysfunction in the community after spinal cord injury: a postal survey in the Republic of Korea. Spinal Cord. 2012;50:303-8.

37. Furusawa K, Tokuhiro A, Sugiyama H, Ikeda A, Tajima F, Genda $\mathrm{E}$, et al. Incidence of symptomatic autonomic dysreflexia varies according to the bowel and bladder management techniques in patients with spinal cord injury. Spinal Cord. 2011;49:49-54.

38. World Medical Association. World Medical Association Declaration of Helsinki: ethical principles for medical research involving human subjects. World Medical Association. 2013;310:2191-4. 